9. Little P, Dorward M, Warner G, et al. Importance of patient pressure and perceived pressure and perceived medical need for investigations, referral, and prescribing in primary care: nested observational study. BMJ 2004: 328(7437): 444

\section{One-dimensional learning: detrimental to the Situational Judgement Test?}

Medical students acquire knowledge, apply theory, and develop skills during their undergraduate training. At medical school, formal teaching sessions tend to focus on the communication between doctor and patient, often with only passing reference to more complex everyday scenarios in which doctors find themselves. There are many situations and diverse interactions demanding effective communication in which the future clinician may be undertrained. These could be with, but are not limited to, members of the patient's family or other healthcare professionals and through different media such as by telephone, email, or with legal personnel. The nature of medical training regarding communication is too one-dimensional. The importance of multiprofessional teamwork is emphasised throughout our training, but opportunities to consolidate this theory in real-life scenarios are all too rare. As a newly qualified doctor or when later holding a position of increased responsibility, a lack of experience of reallife professional interactions may contribute to difficulties with colleagues and lead to increased stress in the workplace. For example it would be helpful to provide strategies for confrontation resolution when in challenging situations. At the other end of the spectrum, introducing students to the art of communicating a referral to another healthcare professional would be beneficial. The focus during training needs to broaden.

Difficult decisions may have to be made in parallel with communicating in unfamiliar settings. Thus the lack of experience of communicating in these situations may affect the development of clinical decisionmaking skills; skills that are indispensable to a competent clinician. For instance, when faced with the uncertainty that often permeates general practice, it is imperative to both arrive at and communicate a well- reasoned, decisive plan of action. This can be complicated further if the setting of the communication is foreign to the clinician. GPs familiar with personal and emotional experiences of these situations will be better equipped to deal with the challenges posed by time, language, cultural, and sensitive issues, and the patient's agenda, while still establishing rapport and trust. The lack of exposure to such scenarios, whether real life or simulated, will surely be to the detriment of future skills as a clinician and also in the short term potentially, in achievement in the Situational Judgement Test.

Jason K Ferdjani,

Brighton and Sussex Medical School, BSMS Teaching Building, University of Sussex,

Brighton, East Sussex, BN1 9PX.

E-mail: J.Ferdjani1@uni.bsms.ac.uk

Amour BU Patel,

Brighton and Sussex Medical School, BSMS Teaching Building, University of Sussex, Brighton, East Sussex, BN1 9PX.

\section{Soram RU Patel,}

Brighton and Sussex Medical School, BSMS Teaching Building, University of Sussex, Brighton, East Sussex, BN1 9PX.

\section{DOI: 10.3399/bjgp12X656766}

\section{Introducing an old teaching tool to general practice}

The term 'doctor' comes from Latin for 'to teach'. In general practice, this can involve teaching patients, medical students, trainees and other health professionals. As part of my medical school rotations, I recently shadowed a doctor who was particularly keen to teach both his patients and medical students. I thought his method of drawing out diagrams for patients was particularly helpful to a patients' understanding of what was happening in their bodies. However, when drawing on paper, it is necessary to show the patient what is being drawn in the correct orientation. This involves positioning oneself next to the patient, that can potentially make some patients uncomfortable. This can be compounded by the doctor inviting medical student(s) to also learn from the drawings at the same time as the patient, creating an unintentional huddle mid-consultation.

I suggest that doctors who are keen to teach in this way consider investing in a whiteboard. Whiteboards have largely replaced traditional chalk and blackboards. Marker pens are used to write on the smooth surfaces of whiteboards, making them easy to erase. A quick search on a popular shopping website revealed that a $60 \times 40$ centimetre whiteboard could be bought for as little as $€ 5$. An investment in a whiteboard may therefore be a way to reduce paper costs in the long term. They may also be more environmentally-friendly than using paper. Importantly, as they can be placed on a part of the wall visible to all, they can be used to teach students and patients simultaneously without unintentional invasion of personal space.

However, careful thought regarding placement of the whiteboard is necessary to avoid introducing a lecturer-student dimension to the already complicated patient-doctor relationship. This may be the case if the doctor has to stand up to write on the board. I suggest placing the board in such a way that the doctor can write on it while sitting down so they remain on the same level as the patient.

Nigel Mabvuure,

Brighton and Sussex Medical School, Phase 2. Audrey Emerton Building, Eastern Road, Brighton, BN2 5BE.

E-mail: N.Mabvuure1auni.bsms.ac.uk

DOI: 10.3399/bjgp12X656775

\section{Are GPs unsympathetic to infertile women?}

Are GPs unsympathetic to infertile women? The National Infertility Awareness Campaign (NIAC) published results from their most recent patient survey on 27th August 2012, based on responses from 456 patients collected in 2011 . While $78 \%$ of responders indicated their GP was sympathetic and helpful, almost 50\% also said they felt their GP lacked the necessary knowledge of infertility and treatment options available to provide an effective service. Clare Lewis-Jones, Chief Executive of NIAC, suggested this could explain why some patients (22\%) with fertility issues perceive their GPs as unsympathetic.'

Dr Clare Gerada, chair of the Royal 
College of General Practitioners said in an interview with the Guardian:

'[GPs] should be supportive because infertility is a hidden pain. That's something we need to do something about and ensure that the profession is sympathetic. ${ }^{2}$

In response to this survey, perhaps it is worth considering again the findings of our recent study. ${ }^{3}$ The interview study asked women and men facing infertility about their experiences of primary care, and then presented the findings to a focus group of GPS.

Patients talked about the importance of being taken seriously and not feeling fobbed off by their GP. The GPs stressed how protocols drive their approach to patients who are potentially infertile but that it was often hard to explain. The GPs also discussed how hard it can be to demonstrate that they are taking patients seriously while also reassuring them that the problem is likely to resolve without treatment. They will often see patients who, with a bit more time, will conceive without need for specialist referrals.

Some GPs will rarely see couples with infertility, whereas others will see a reasonable number. The GPs in our study agreed offering support and reassurance was an appropriate role for GPs. It was suggested that the most effective way of communicating this was drawing up an action plan and flagging the patients' notes so their treatment can be followed up, or questions raised at their next consultation. These simple steps could go a long way to reassuring the patient that their concerns are being taken seriously.

Lisa Hinton,
Senior Qualitative Researcher, Health Experiences Research Group, Department of Primary Health Care Sciences, University of Oxford, Oxford.

E-mail: lisa.hintonaphc.ox.ac.uk

\section{REFERENCES}

1. Lewis-Jones C. Infertility and IVF: results of patient survey show educating GPS is the best way forward. London: BioNews, 2012. http://wnw. bionews.org.uk/page_171282.asp laccessed 14 Sep 2012).

2. Campbell D. GPs unsympathetic to infertile women, study finds. Guardian 2012; 27 Aug: http://www.guardian.co.uk/society/2012/ aug/27/gps-unsympathetic-infertile-womenstudy? INTCMP=SRCH (accessed 14 Sep 2012).

3. Hinton L, Kurinczuk JJ, Ziebland S. Reassured or fobbed off? Perspectives on infertility consultations in primary care: a qualitative study. Br J Gen Pract 2012; DOI: 10.3399/bjgp12X649133.

DOI: 10.3399/bjgp12X656784 